\title{
Improvements and Analysis of Nonlinear Parallel Behavioral Models
}

\author{
Daniel D. Silveira, ${ }^{1}$ Pere LI. Gilabert, ${ }^{2}$ Pedro M. Lavrador, ${ }^{3}$ José C. Pedro, ${ }^{3}$ \\ Michael Gadringer, ${ }^{4}$ Gabriel Montoro, ${ }^{2}$ Eduard Bertran, ${ }^{2}$ Gottfried Magerl ${ }^{4}$ \\ ${ }^{1}$ R\&D Department, Linear Equipamentos Eletronicos, Santa Rita do Sapucai, MG, Brazil \\ 2 Department of Signal Theory and Communications, Technical University of Catalonia, \\ C/Esteve Terradas, 7-08860 Castelldefels, Barcelona, Spain \\ ${ }^{3}$ Instituto de Telecommunicacoes, Universidade de Aveiro, 3810-193 Aveiro, Portugal \\ ${ }^{4}$ Institute of Electrical Measurements and Circuit Design, Vienna University of Technology, \\ Gusshausstrasse 25/354, A-1040 Vienna, Austria
}

Received 1 December 2008; accepted 28 March 2009

\begin{abstract}
This article performs an analysis of current limitations regarding the extraction of parallel behavioral models to reproduce the power amplifier (PA) nonlinear behavior and its dynamics. To overcome these limitations, a general preprocessing block that clearly improves the identification capabilities shown by classical parallel structures is proposed. It follows the principle of separating both static and dynamic nonlinear behavior of the PA to obtain a better identification performance. A comparison with common parallel configurations using linear estimation is performed, to highlight the benefits of using the preprocessing structure. Furthermore, a new nonlinear parallel structure using sub-band filtering techniques is also proposed. For the models extraction and comparison, four types of noise-free simulated data presenting different levels of nonlinearities and memory, as well as a measured signal obtained from a laboratory amplifier have been considered. (C) 2009 Wiley Periodicals, Inc. Int J RF and Microwave CAE 19: 615-626, 2009.
\end{abstract}

Keywords: behavioral; microwave power amplifiers; modeling; nonlinear systems; estimation

\section{INTRODUCTION}

Behavioral models do not need an a priori knowledge of the power amplifier (PA) internal composition. For that reason, they are also known as black-box models. Their extraction relies on a set of input-output observations. Therefore, their accuracy is highly sensitive to the adopted model structure and the parameter extraction procedure. Behavioral modeling finds applications in the field of system simulation and PA

Correspondence to: D. D. Silveira; e-mail: danieldsilveira@ gmail.com

DOI 10.1002/mmce.20385

Published online 13 July 2009 in Wiley InterScience (www. interscience.wiley.com). linearization, where it is essential to trade-off accuracy and computational efficiency.

A common strategy to obtain more accurate behavioral models consists of adding several structures in parallel. Parallel models have been successfully used in nonlinear system identification in different areas using time or frequency-domain data, as in [1$3]$. The advantage of parallel models regards the possibility of integrating several structures in different branches, providing flexibility in the design. The convergence is guaranteed because the next branch is conformed to the residue left by the previous one. The disadvantage is that parallel models are very sensitive to noise if too many paths are added. Consequently, a proper selection of the paths and the order 


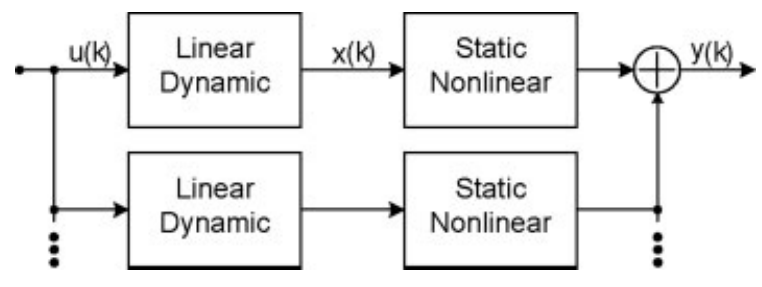

Figure 1. Parallel Wiener model.

of the nonlinearity should be made to assure low noise and good convergence [4].

An example of a parallel model that uses time-domain data for its extraction is reported in $[1,3]$ and shown in Figure 1, where input/output signals are functions of the integer $k$, denoting sampled time-domain signals. The estimation procedure for this case can be summarized as follows: In a first approach, a filter (linear time invariant block) is estimated between input signal $u(k)$ and output signal $y(k)$; then an intermediate signal $x(k)$ is obtained as the result of filtering the input signal with the previously obtained filter; and finally, a polynomial is estimated by means of this intermediate signal and the output signal. The error generated by this two-step linear least-squares (LS) estimation is then captured by the next branch of the parallel structure, and so on until no significant improvement in the entire model performance can be observed.

Another example is the modified parallel Hammerstein $(\mathrm{PH})[5,6]$, which uses one filter for each nonlinear order. Its structure allows the determination of the equivalent main diagonals of the Volterra kernels in one step, being an accurate and interesting alternative.

A further accuracy improvement in parallel modeling is possible by considering the use of a different structure (than the one used for the first branch) on the second branch, capable of estimating the remains of dynamics that could not be captured by the first branch. An example of this structure can be found for frequency domain in [2], and it is shown in Figure 2. In these models, a path is designed for the linear part of the signal (filter $H_{\mathrm{L} 1}(f)$ ) and another path for the nonlinear one, namely amplitude/amplitude and amplitude/phase (AM/AM and $\mathrm{AM} / \mathrm{PM})$ nonlinearity and filter $H_{\mathrm{L} 2}(f)$. Treating the residue with another structure, better results were achieved in comparison with a model that presents branches with the same structure. However, an advanced strategy for the initial path estimation and the

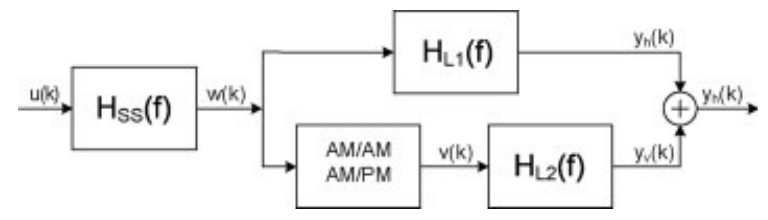

Figure 2. Polyspectral model. correct selection of the paths to be added to the parallel model are crucial to obtain even better modeling results, as it will be shown in the following sections.

\section{PREPROCESSOR FOR PA LINEAR ESTIMATION}

The linear LS estimation combines very interesting characteristics among them. A one shot solution can be computed analytically, a recursive formulation is possible, and it can be used in real time [7]. All these characteristics make LS a widely used estimator.

Nevertheless, it treats linear and nonlinear components of the data in the same way, and to correct this problem applying weighted LS for every particular input signal is a cumbersome task. Focusing in the particular case of PA modeling, the linear components present much more power than the nonlinear ones. Normally, the distortion is at least $30 \mathrm{~dB}$ under the carrier. If models are estimated directly from input/output sets of data, both linear and nonlinear behavior will be modeled together and thus the linear part of the signal will appear as high amplitude noise for the nonlinear part within the estimation. This unwanted situation can be avoided by removing the linear part of the signal. This could be accomplished by fitting a best linear approximation between input/ output data, in a similar approach to the one described for polyspectral models [2].

Furthermore, the nonlinear dynamic part of the PA output, $y_{\mathrm{dyn}}(k)$, has usually much less power than its associated static or memoryless components, $y_{\mathrm{NL}}(k)$. Therefore, we now go a step further, and propose a parallel model consisting of a first preprocessing branch, that represent the memoryless nonlinearity $(\mathrm{NL})$, and subsequent branches, responsible for modeling the remaining of the output signal that has not been identified by the first branch.

The estimation procedure for the extraction of the model with preprocessing can be summarized as follows (see Fig. 3):

1. Extract the memoryless nonlinear model (NL block) by means of the measured input $\left(u_{\text {meas }}(k)\right)$ and output $\left(y_{\text {meas }}(k)\right)$ data;

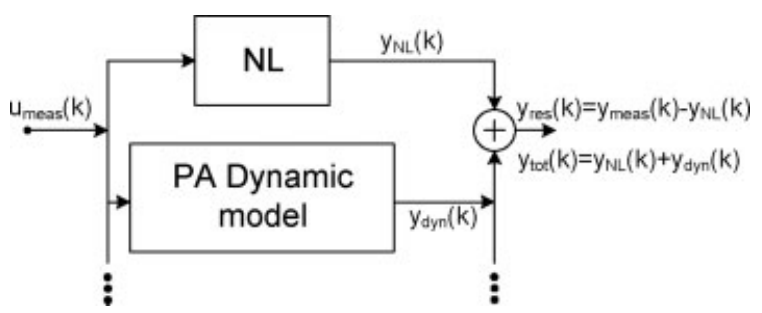

Figure 3. Preprocessing for a dynamic PA model. 


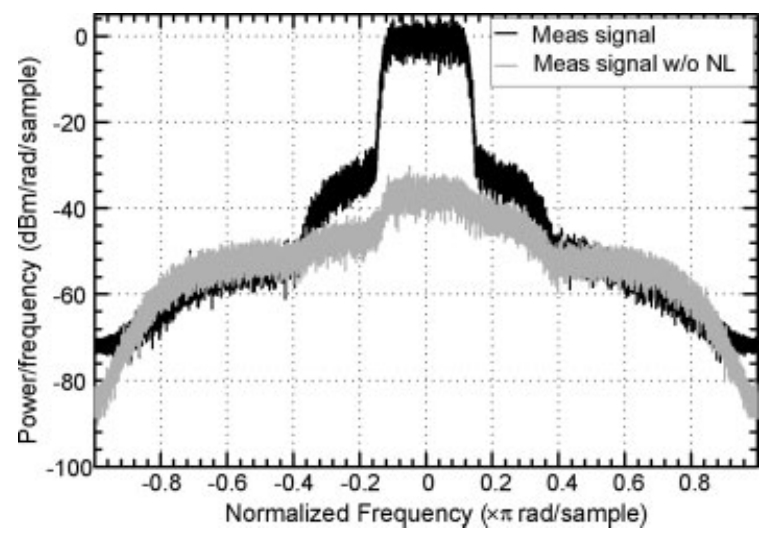

Figure 4. Residue at the output of the NL branch (Meas Signal w/o NL).

2. Calculate the residual signal $y_{\text {res }}(k)$ defined as the subtraction between the measured output $y_{\text {meas }}(k)$ and the output of the memoryless nonlinear model $y_{\mathrm{NL}}(k)$;

3. Finally, parameterize the dynamic model using the input signal $u_{\text {meas }}(k)$ and the residual signal $y_{\text {res }}(k)$.

The output of the overall model is the sum of the outputs of both branches, NL $\left(y_{\mathrm{NL}}(k)\right)$ and PA dynamic model $\left(y_{\text {dyn }}(k)\right)$. The process can still be continued, if other residues are calculated and suitable models are used.

Explaining further, the NL branch (preprocessing) removes all the static nonlinearity of the signal, or the noise for the identification of nonlinear distortion parameters, allowing a more accurate identification of the dynamic behavior (PA dynamic model branch). An example of a measured signal without the nonlinear static components (residue for the next estimation, $\left.y_{\text {res }}(k)\right)$ is displayed in Figure 4.

The NL branch can be estimated as the best memoryless approximator, in a mean-square error sense

$$
\min \left\{\left|\sum_{k=0}^{K} y_{\text {meas }}(k)-y_{\mathrm{NL}}(k)\right|^{2}\right\}
$$

where $y_{\text {meas }}(k)$ is the measured PA output response and $y_{\mathrm{NL}}(k)$ are the static or memoryless components.

This allows the computation of the residual dynamic output component $y_{\text {dyn }}(k)$

$$
y_{\text {dyn }}(k)=y_{\text {meas }}(k)-y_{\mathrm{NL}}(k) .
$$

When this best nonlinear memoryless approximator, $y_{\mathrm{NL}}(k)$, has a polynomial form, its coefficients can be easily obtained from a linear LS estimation derived from

$$
\min \left\{\left|\sum_{k=0}^{K} y_{\text {meas }}(k)-\sum_{p=1}^{P} a_{p} u^{P}(k)\right|^{2}\right\} .
$$

where $a_{p}$ is the power series coefficient $a$ of order $p$, and $u(k)$ is the input signal.

A similar approach was suggested in [3], but limited to build the nonlinearity with the two-tone AM/ AM-AM/PM.

Alike in [2], it is possible to replace the memoryless nonlinearity (NL branch, in Fig. 3) by a filter, in order to remove linear PA dynamics to proceed with the identification of the nonlinear part in further branches. Then, we could reformulate (1)-(3) as

$$
\min \left\{\left|\sum_{k=0}^{K} y_{\text {meas }}(k)-y_{\mathrm{L}}(k)\right|^{2}\right\}
$$

where $y_{\mathrm{L}}(k)$ is the best linear approximator. This allows the computation of the residual static nonlinear output component as

$$
y_{\mathrm{NL}}(k)=y_{\text {meas }}(k)-y_{\mathrm{L}}(k) .
$$

When the best linear approximator is modeled as an FIR filter, its coefficients can be easily obtained from a linear LS estimation derived from

$$
\min \left\{\left|\sum_{k=0}^{K} y_{\text {meas }}(k)-\sum_{\tau=0}^{M-1} h(\tau) u(k-\tau)\right|^{2}\right\} .
$$

where $h(\tau)$ is the impulse response of a filter with memory depth $M$.

However, the identification performance achieved with this solution, applied in [8], is too dependent on the number of coefficients used to describe the filter and thus it loses generality in the comparison. Moreover, as pointed out in [9], the procedures to extend the model were not given in [8]. Based on these difficulties, the preprocessor technique with the filter replacing the NL branch will not be further applied in this article. The studies will follow applying the preprocessor containing the NL branch and the PA dynamic model branch (Fig. 3).

To verify the identification performance achieved when using this preprocessing technique, sets of simulated and measured input/output data were built. The generation of these data is explained in detail in the next section.

Further on, several structures for PA modeling (with and without preprocessing) were extracted from 
these data and the identification accuracy of all estimated models is compared.

\section{GENERATION OF PA INPUT/OUTPUT DATA}

Five different groups of input/output data sets have been considered for the behavioral modeling extraction and validation. Four groups of four input/output data sets were obtained on a simulated amplifier designed to present controlled memory effects as shown in Figure 5, and one group of two input/output sets is relative to a measured input/output WiMAX signal on an LDMOS PA.

The 16 input/output sets of simulated data corresponding to different memory situations and nonlinear characteristics considered for the comparison were as follows: (i) memoryless, (ii) linear memory, (iii) nonlinear memory, and (iv) linear and nonlinear memory simultaneously. The simulated data were obtained from a PA designed to handle a WCDMA signal at $1.9 \mathrm{GHz}$, operating with an input back-off (IBO) of $2 \mathrm{~dB}$. To show the model capabilities for dealing with nonlinear dynamic systems, the output bias network was designed to deliberately introduce (or not) a reasonable amount of nonlinear or linear memory. The schematic of the circuit used to obtain these data is depicted in Figure 5. By introducing (or not) the highlighted subcircuits 1 and 2 ( $\mathrm{SC} 1$ and $\mathrm{SC} 2$ ), the different types of data (prioritizing memory or nonlinearities) can be generated on demand.

The large bias inductor $(6.18 \mathrm{uH})$ shown in Figure 5 is responsible for the nonlinear long-term memory effects. The inclusion of subcircuit 1 (SC1, in Fig. 5) (almost) completely eliminates the impact of the bias inductor on the baseband signal bandwidth creating this way a system without significant nonlinear memory effects. The parallel LC of subcircuit 2 (SC2) is designed to have a strong slope at the carrier frequency and thus to present high frequency response variations near these frequencies. So, the inclusion (or not) of SC2 determines the presence of linear memory at the output of the considered circuit.

To illustrate the dynamic behavior of the different circuit configurations, Figure 6 presents the instantaneous gain variation with the instantaneous input power using a two tone signal of $1 \mathrm{MHz}$ separation (referred in following Tables as noM-Memoryless, LM-linear memory, NLM-nonlinear memory, LM/ NLM-linear memory, and nonlinear memory). The fifth group of measured sets of input/output data was obtained from a LDMOS Wimax amplifier at $3.5 \mathrm{GHz}$ operating at $2 \mathrm{~dB} \mathrm{IBO}$, and $3.5 \mathrm{MHz}$ RF bandwidth,

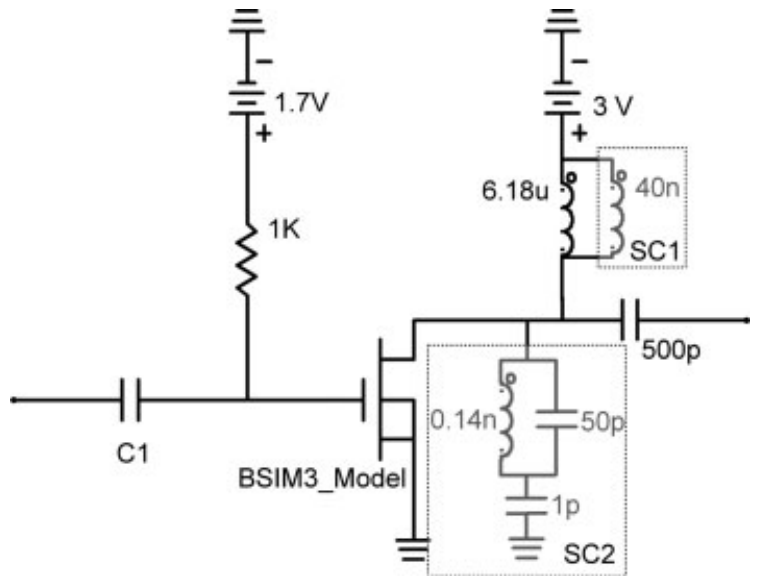

Figure 5. Schematic representation of the circuit used to generate the different types of memory.

using a modified bias Tee to introduce additional memory in measurement results [10]. The AM/AM and AM/PM characteristics of this modified amplifier measured signal are displayed in Figure 7. The measurement setup covered intermodulation distortion bands of up to seventh order. The input/output power spectral density (PSD) is shown in Figure 8. This signal is referred in the following tables as Meas.

Based on these sets of data, tables of comparisons were built and some conclusions were drawn as shown in the next section.

\section{MODEL EXTRACTION AND RESULTS}

A first comparison among behavioral models frequently used in the literature, namely ordinary baseband power series [11], Wiener, parallel Wiener (PW), and modified parallel Hammerstein models $(\mathrm{PH})$, was done using the simulated and measured set of data, and summarized in Table I. The figure of merit used to characterize model's accuracy was the normalized mean square error (NMSE) [2]. Models were extracted considering a seventh order baseband polynomial and a maximum of three delay taps. Although a similar comparison was previously reported in [6], we will use the results obtained by the comparison presented in this article to support the performance of the new techniques presented. Observing results in Table I, we can see that the parallel Wiener with two branches shows practically the same NMSE than a single Wiener model for all different type of data. No significant accuracy improvement, measured in terms of NMSE, is appreciated. The inclusion of additional branches in the model would not be justified, because it would be modeling 

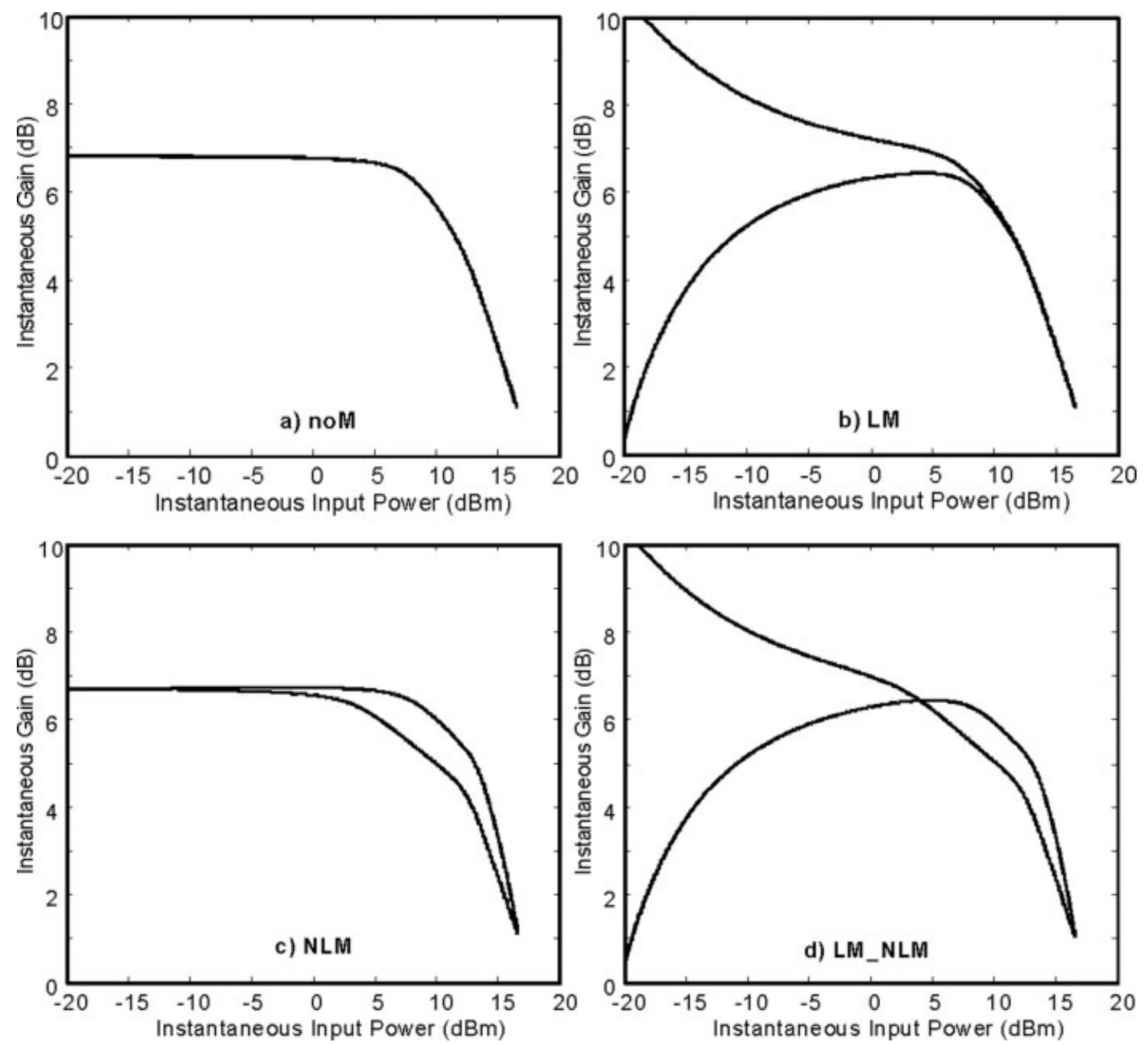

Figure 6. Instantaneous input/output gain simulated with a two tone signal for the different circuit configurations. (a) Memoryless; (b) with linear memory; (c) with nonlinear memory; and (d) with linear and nonlinear memory.
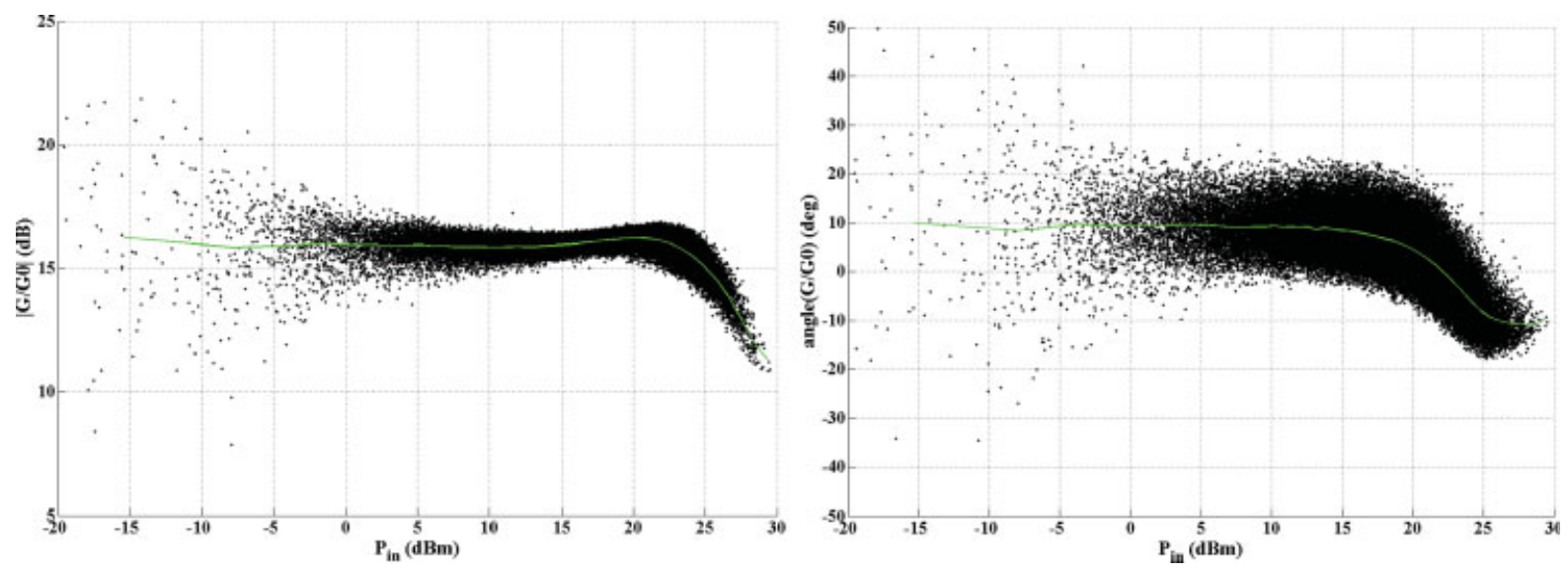

Figure 7. $\mathrm{AM} / \mathrm{AM}$ and $\mathrm{AM} / \mathrm{PM}$ characteristics of the modified bias Tee amplifier measured signal used for modeling. [Color figure can be viewed in the online issue, which is available at www.interscience.wiley.com.] 


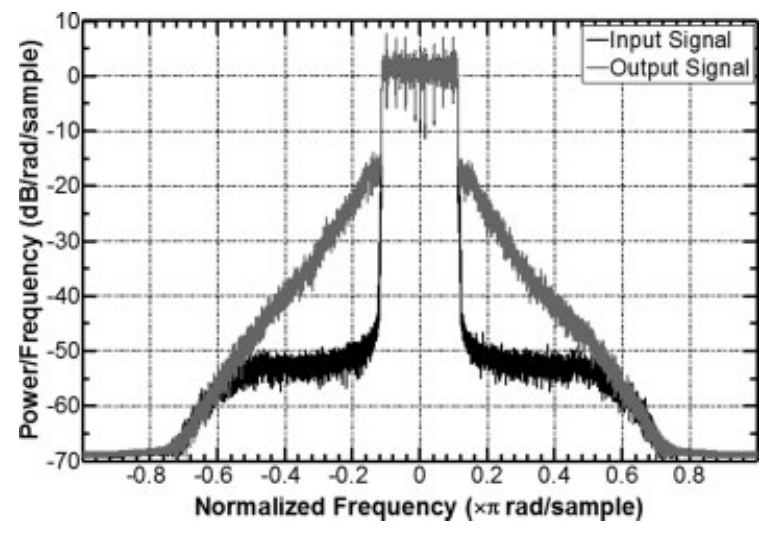

Figure 8. PSD of the modified bias Tee amplifier input/ output measured signal.

noise and thus not contributing to the final estimation results. The PH model shows the best NMSE results, because the equivalent Volterra kernels coefficients were estimated in one step. Notice that NMSE values obtained when considering the measured data are considerably poorer than when considering the noisefree simulated data sets, because measured data were affected by noise and possible synchronization imperfections, and collected from a PA operating in deep compression. Taking into account these results one may conclude that the addition of parallel replicas of the same model structure does not significantly contribute to obtain a more accurate identification performance.

To highlight the advantages of the general preprocessing technique presented in Figure 3, we particularize the general dynamic PA model with a reduced Volterra series using a Wiener-Bose structure (WBose) [12], as shown in Figure 9.

This behavioral model with preprocessing is composed of a nonlinear memoryless block, implemented with a look-up table (LUT) to avoid the dependency on the polynomial order, and the WBose block. Each block is explained in details below:

- The LUT is determined dividing the measured data in AM/AM and AM/PM conversion curves in slices, which are dependent of the

TABLE I. NMSE Summary—Simulated and Measured Signal

\begin{tabular}{lccccc}
\hline Model & $\begin{array}{c}\text { LM/NLM } \\
(\mathrm{dB})\end{array}$ & $\begin{array}{c}\text { LM } \\
(\mathrm{dB})\end{array}$ & $\begin{array}{c}\text { NLM } \\
(\mathrm{dB})\end{array}$ & $\begin{array}{c}\text { noM } \\
(\mathrm{dB})\end{array}$ & $\begin{array}{c}\text { Meas } \\
(\mathrm{dB})\end{array}$ \\
\hline Poly & -34 & -36 & -36 & -40 & -21 \\
Wiener & -37 & -40 & -36 & -40 & -21 \\
PW & -37 & -40 & -36 & -40 & -21 \\
PH & -37 & -40 & -37 & -41 & -22 \\
\hline
\end{tabular}

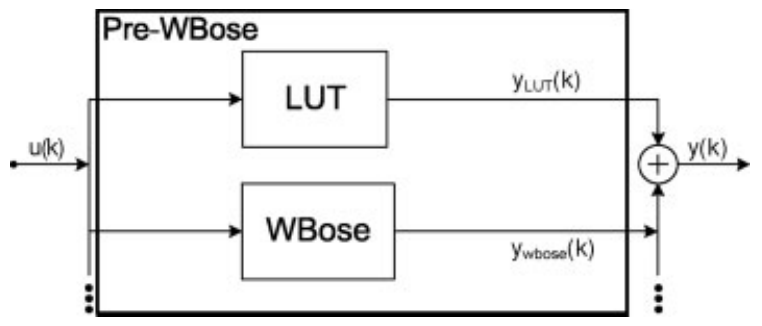

Figure 9. Proposed initial configuration for the estimation of PA behavioral models.

input power and the number of points involved. Figure 10 shows a histogram that contains the number of points taken into account to construct the corresponding slice. This histogram shows a nonuniform distribution of the points, dependent of the instantaneous input power. Then, AM/AM and AM/PM conversion curves of the signal are determined directly from the mean value of the slices, as it is depicted in Figure 11. From these curves, the LUT is parameterized;

- The WBose block was modeled with only three delay taps. Moreover, to reduce the complexity of the model, the pruning technique proposed in [13] has been used. It was originally derived until 5th order baseband Volterra series, and was extended to the 7th order to be used in this work. This derivation was not published, but can be sent by the author upon request. The pruned kernels are represented in (7).

$y_{\text {WBose }}(k)=\sum_{p} \sum_{r_{1}=0}^{M-1} \cdots \sum_{r_{p}=0}^{M-1} \mathrm{hr}_{p}\left(\tau_{1}, \ldots, \tau_{p}\right) \prod_{j=1}^{p} u\left(k-\tau_{j}\right)$.

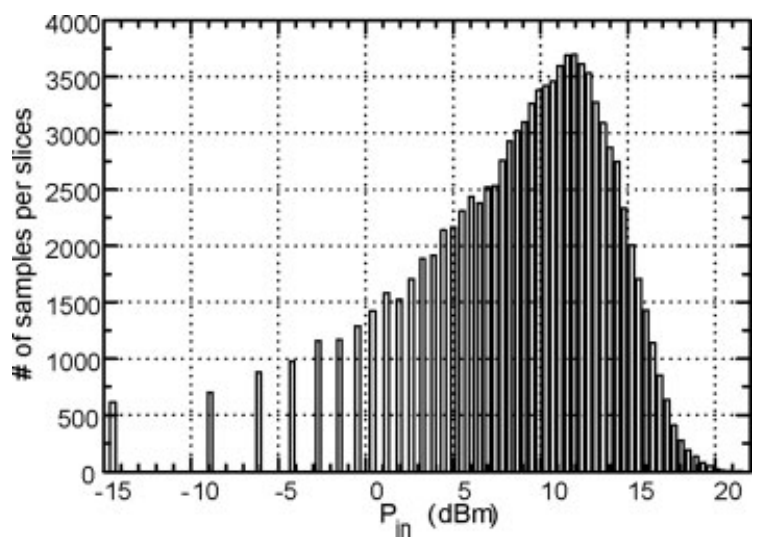

Figure 10. Histogram of the AM/AM nonparametric estimation. 


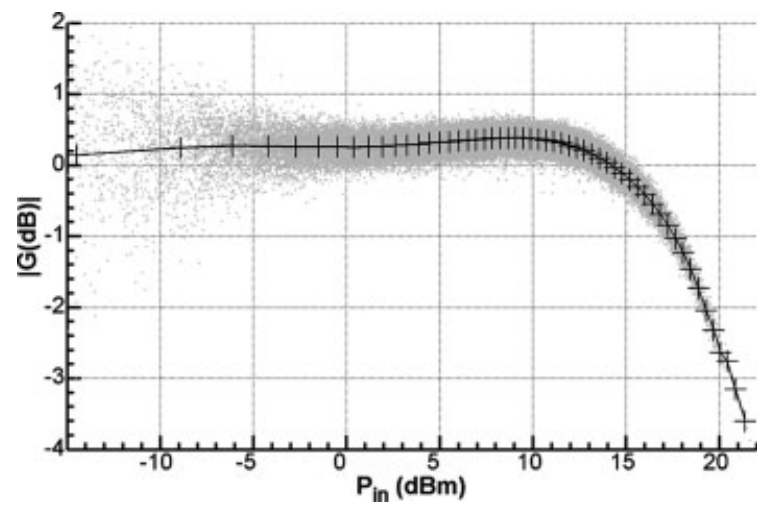

Figure 11. $A M / A M$ preprocessing (AM/PM is similar).

where $\operatorname{hr}_{p}\left(\tau_{1}, \ldots, \tau_{p}\right)$ is the reduced Volterra kernel of order $p$, with $M$ memory length, and $u(k)$ as input signal.

The output of the overall model is the sum of the outputs of both branches, as it is depicted in Figure 9 and shown by (8).

$$
\begin{aligned}
y(k)=\operatorname{LUT}[u(k)]+\sum_{p} \sum_{\tau_{1}=0}^{M-1} \cdots & \sum_{r_{p}=0}^{M-1} h r_{p}\left(\tau_{1}, \ldots, \tau_{p}\right) \\
& \times \prod_{j=1}^{p} u\left(k-\tau_{j}\right) .
\end{aligned}
$$

where LUT[·] is the LUT operator.

To avail the effectiveness of this method, Table II shows the identification results for a memoryless nonlinearity (LUT alone); a $\mathrm{PH}$ with preprocessing (Pre-PH); a WBose model without preprocessing (WBose); and a WBose model with preprocessing (Pre-WBose). The WBose model presented the best results for models without preprocessing and for signals with memory, comparing Tables I and II. This result was expected because a reduced Volterra model includes more memory effects in its structure than the other models. Only when the signal has no memory (noM, Table II), the NMSE (dB) of the LUT was better than the WBose model. This effect is known as noise modeling $[4,7]$. Once there was no memory in the system to be identified, a very complete model as a reduced-Volterra approximation (in terms of memory effects) was estimated based on noise, delivering worse results than a static nonlinear model.

Unlike the use of parallel replicas (see results in Table I), the use of the proposed preprocessing technique improves the identification results achieved by a single dynamic model without any kind of preprocessing. The results of the Pre-PH were better than a WBose for signals containing only linear memory, and the Pre-WBose has better results than all other models. Therefore, this technique offers the possibility to increase the identification accuracy with only a slight change in the estimation procedure. Removing first the nonlinear static part of the signal before applying the WBose model estimation has improved the accuracy, as the nonlinear static part of the signal has much higher amplitude than the dynamic part of the signal. In single one-step estimation, both parts would be treated the same way by the LS estimator, as in the case without preprocessing.

Results also show that further improvements for this modeling technique using LUT and WBose are hard to achieve. The solution found was the use of different structures at the subsequent branches, which could improve the NMSE results, like the sub-band structures explained in the next section. In the following, the data used to compare and validate the proposed architectures and techniques will be the measured data obtained from the modified bias Tee amplifier.

\section{ESTIMATING MEMORY EFFECTS WITH SUB-BAND STRUCTURES}

An RF power amplifier has a complex structure, presenting many kinds of memory. They can be classified as [14]:

- Low frequency ( $\mathrm{kHz}$ to $\mathrm{MHz}$ ): thermal effects, trapping effects, biasing circuits, AGC loops;

- High frequency $(\mathrm{GHz})$ : transistor (transit time and reactance parasitics), matching networks (group delay).

These memories are mixed together in the PA (nonlinear coupled), and the problem of estimating behavioral models becomes very difficult [15].

Models capable of identifying memory at different signal rates can improve the identification performance, which can be accomplished using parallel subband filtering techniques. This is a powerful method to design very large order FIR filters, operating at a

TABLE II. NMSE Summary of Ordinary Models and Models with Preprocessing-Simulated and Measured Signal

\begin{tabular}{lccccc}
\hline Model & $\begin{array}{c}\text { LM/NLM } \\
(\mathrm{dB})\end{array}$ & $\begin{array}{c}\text { LM } \\
(\mathrm{dB})\end{array}$ & $\begin{array}{c}\text { NLM } \\
(\mathrm{dB})\end{array}$ & $\begin{array}{c}\text { noM } \\
(\mathrm{dB})\end{array}$ & $\begin{array}{c}\text { Meas } \\
(\mathrm{dB})\end{array}$ \\
\hline LUT & -35 & -38 & -37 & -60 & -21 \\
Pre-PH & -38 & -54 & -38 & -64 & -22 \\
WBose & -42 & -42 & -42 & -42 & -23 \\
Pre-WBose & -44 & -56 & -44 & -65 & -23 \\
\hline
\end{tabular}




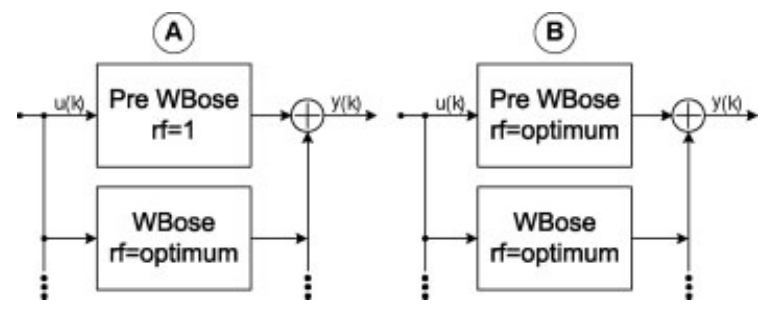

Figure 12. Proposed parallel models with resampling configuration for the estimation of PA behavioral models.

high speed, with smaller filters, operating at slower speed, reducing computational complexity, as shown in $[16,17]$. In this technique, the input signal is decimated into different rates, filtered for each branch, and later interpolated with the respective branch rate to form the output signal. With smaller filters, the matrix to be inverted in LS estimation process is also smaller. An example of baseband digital predistortion using this technique if found in [17]. A system identification problem, where sub-band neural networks were used to recover audio signals is shown in [18]. It was concluded that sub-band adaptive filters have better performance for highly correlated input signals (also the case for amplifier identification) than fullband adaptive filters. Also in [18] it was proven that, for sub-band input signals, the eigenvalue spread of the sub-band signal will be smaller or equal the eigenvalue spread of the full signal, which guarantees a better condition number of the Hessian matrix used in the LS estimation.

The sub-band principle was applied in an innovative parallel model, with three variants:

- Parallel model with resampling A-PMRA and parallel model with resampling B-PMRB, presented in Figure 12;

- Parallel model with resampling using simulated annealing-PMRSA, displayed in Figure 13.

This model is capable of estimating different kinds of memories, mixing it with nonlinearities in an effective way, increasing the model accuracy, and representing a PA as close as possible, as will be shown.

The overall model output $y(k)$ of the PMRA, PMRB, and PMRSA is obtained by the sum of all responses at each branch

$$
\begin{aligned}
y(k)=\operatorname{LUT}[u(k)]+\sum_{p} \sum_{r_{1}=0}^{M-1} \cdots \sum_{r_{p}=0}^{M-1} h \mathrm{r}_{p(b)} \\
\times\left(r \mathrm{f}_{b} \cdot \tau_{1}, \ldots, r \mathrm{f}_{b} \cdot \tau_{p}\right) \prod_{j=1}^{p} u\left(k-r \mathrm{f}_{b} \cdot \tau_{j}\right) .
\end{aligned}
$$

where $\mathrm{rf}_{b}$ is the resampling factor $r \mathrm{f}$ of the branch $b$ used in the model.

The variants PMRA and PMRB will differ in the way that a resampling factor $\left(r_{\mathrm{f}}\right)$ [12], which allows each branch to be estimated in a different rate, is optimized. The model implemented with the $r_{\mathrm{f}}$ can re-produce copies of the nonlinear FIR in the entire spectrum, and a better resolution for the same memory depth inside the chosen sub-band frequency band is obtained. This degree of freedom for $r_{\mathrm{f}}$ can be optimized in several model extraction and evaluation, as it has been done in this work. After that, the residue is obtained, and the process is repeated for the subsequent branches until no significant improvement is achieved. Therefore, the model is composed of branches optimized for different rates having improved identification capabilities.

The structure of PMRA allows $r_{\mathrm{f}}$ optimization only after the first branch. This means that the first branch has a fixed $r_{\mathrm{f}}$ of 1 , thus doing a "blind" first estimation, and then an optimization is performed for the other branches considering different values of $r_{\mathrm{f}}$.

Using this technique and using the input/output measured data obtained from the modified bias Tee amplifier, the first branch of the PMRA was calculated, and presented an NMSE of $-23 \mathrm{~dB}$, as displayed in Table III, under column First branch $r_{\mathrm{f}}=$ 1. The $r_{\mathrm{f}}$ was optimized for each further branch. This was accomplished varying $r_{\mathrm{f}}$ and performing estimation for this point, recording the results, building a graph as Figure 14, and locating the best NMSE (dB) result as a function of $r_{\mathrm{f}}$. As the overall result is the sum of the output signal from each branch, that $r_{\mathrm{f}}$ that resulted in the lowest NMSE was selected. Figure 14 shows the results of the $r_{\mathrm{f}}$ optimization curves for branches from 2 to 5 and considering $r_{\mathrm{f}}=1$ in the first branch. The final NMSE was the sum of the NMSE of all branches $(-29 \mathrm{~dB}$, as displayed in Table III). As expected, additional branches presented

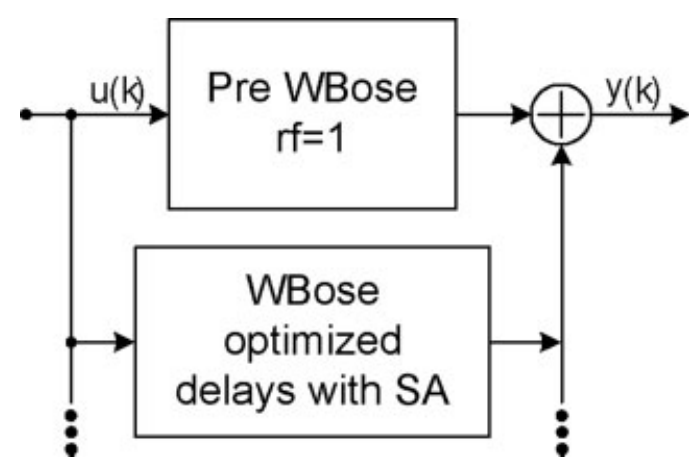

Figure 13. Variation with simulated annealing optimization for the subsequent branches-PMRSA. 
TABLE III. NMSE, $r_{\mathrm{f}}$ and Optimal Delays Results

\begin{tabular}{lrrrrcc}
\hline \multicolumn{3}{c}{$\begin{array}{c}\text { PMRA First branch } \\
r_{\mathrm{f}}=1\end{array}$} & \multicolumn{3}{c}{$\begin{array}{c}\text { PMRB Optim. for all } \\
\text { Branches }\end{array}$} & $\begin{array}{c}\text { PMRSA } \\
\text { Optim. } \\
\text { with SA }\end{array}$ \\
\hline \multicolumn{4}{c}{ NMSE } & \multicolumn{3}{c}{ NMSE } \\
Branch & $r_{\mathrm{f}}$ & $(\mathrm{dB})$ & $r_{\mathrm{f}}$ & $(\mathrm{dB})$ & Delays & $\begin{array}{c}\text { NMSE } \\
(\mathrm{dB})\end{array}$ \\
\hline 1 & 1 & -23 & 9 & -24 & {$\left[\begin{array}{lll}1 & 2 & 3\end{array}\right]$} & -23 \\
2 & 19 & -3 & 12 & -2 & {$\left[\begin{array}{lll}1 & 8 & 24\end{array}\right]$} & -4 \\
3 & 13 & -1 & 4 & -1 & {$\left[\begin{array}{lll}1 & 8 & 6\end{array}\right]$} & -0.5 \\
4 & 30 & -1 & 39 & -1 & {$\left[\begin{array}{lll}1 & 20 & 29\end{array}\right]$} & -0.5 \\
5 & 1 & -1 & 30 & -1 & - & - \\
Model & & -29 & & -29 & & -28 \\
\hline
\end{tabular}

always less contribution than the previous ones, indicating a reduction of unmodeled memory effects.

The PMRB begins the rate optimization already in the first branch, which presented an NMSE of $-24 \mathrm{~dB}$ and $r_{\mathrm{f}}$ of 9 , results listed also in Table III, under Optim. for all Branches column. Figure 15 shows curves for the $r_{\mathrm{f}}$ optimization already in the first branch, and Figure 16 for the remaining ones. No significant changes were noted after 50, so $r_{\mathrm{f}}$ iterations were limited up to this value.

Although the PMRB presented a better NMSE figure than the PMRA in the initial branch, the final optimization results were practically the same. So, initial conditions (or the $r_{\mathrm{f}}$ optimization already in the first branch) were considered good for both cases. What is significant is that the use of the resampling factor together with parallel models has improved final results in $\sim 6 \mathrm{~dB}$ NMSE in comparison with results shown in Table II (results obtained using the measured signal). The models here presented have an increased complexity in comparison with previous models, but the accuracy is clearly better.

A third variant of model optimization was tested, using the heuristic search algorithm named simulated

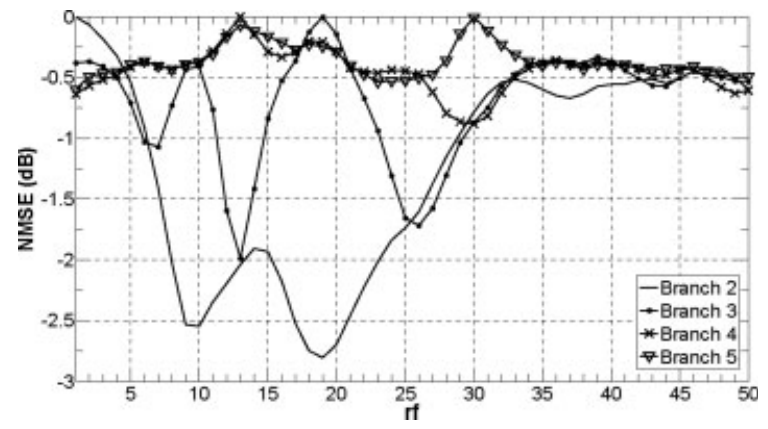

Figure 14. Results for optimization of the PMRA at remaining branches. The best resampling factors were 19 , $13,30,1$ for branches 2 to 5 , respectively.

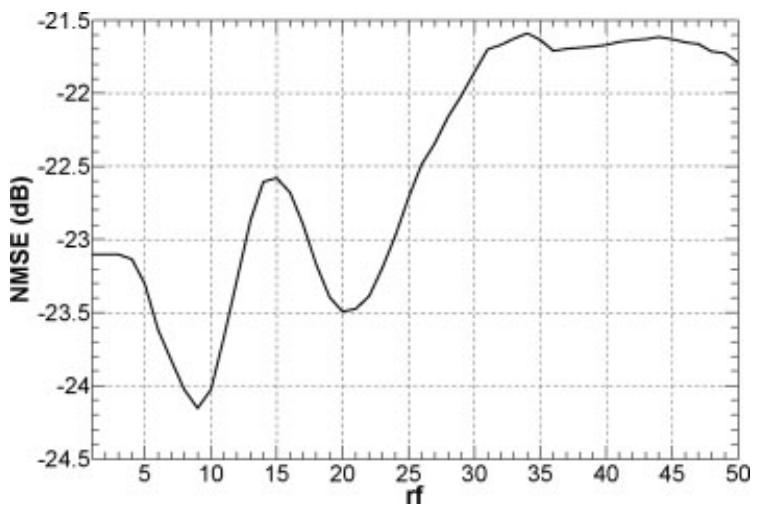

Figure 15. Results for optimization of the PMRB at the first branch. The best resampling factor was found at position 9 .

annealing (SA)-PMRSA. This algorithm is used to find the best sparse delays contributing at each branch to the identification of the behavioral model of the PA, as displayed in Figure 13. This nonlinear search technique has been used in the extraction of baseband behavioral models [19]. It searches for the best configuration of delays to improve the identification accuracy. The results achieved in terms of NMSE using this method are also listed in Table III. It can be observed that already in the second branch, the PMRSA captures a considerable part of the residue, having a faster convergence than the PMRA and PMRB. However, the total NMSE figure is slightly worse than PMRA and PRMB. Nevertheless, the NMSE figure is improved in comparison to the use of a single branch for the identification. The absence of the fifth branch is due to the noise modeling of this branch, which does not contribute to the final results.

At last, PMRA was also tested considering all different sets of simulated data. The obtained results were compared with the other modeling techniques presented in Table II and repeated here for conven-

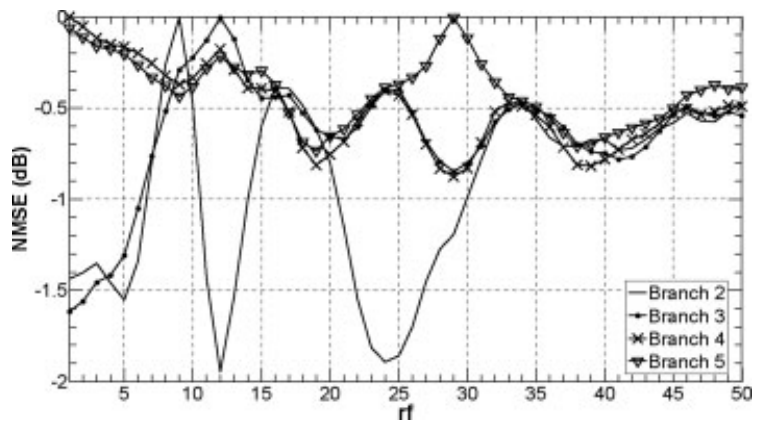

Figure 16. Results for optimization of the PMRB at remaining branches. The best resampling factors were 12 , $4,39,30$ for branches 2 to 5 , respectively. 
ience in Table IV. For memoryless and linear memory systems, no additional branches were necessary, and results were better than $-55 \mathrm{~dB}$ NMSE. For systems presenting nonlinear memory, additional branches were necessary to improve final results, staying around $-48 \mathrm{~dB}$ NMSE. An unmodeled residue was initially present and minimized by subsequent branches.

Also improvements comparing with other modeling techniques were evident: $4 \mathrm{~dB}$ NMSE for simulated signals, and $6 \mathrm{~dB}$ NMSE for measured signals, when compared with Pre-WBose (without parallel additional branches). Comparing with a memoryless model (LUT), improvements were from 5 to $18 \mathrm{~dB}$ NMSE.

To highlight the spectral improvements of this technique, Figure 17 shows the measured output signal and the residues of the model with preprocessing and subsampling parallel branches (PMRA, PreWbose par) and the model with only preprocessing (Pre-Wbose) - the NMSE results are listed in Table IV, in column Meas. The in-band residue improvements of the model using subsampling techniques are clearly seen in this figure.

\section{CONCLUSIONS}

A general preprocessing technique for PA behavioral modeling was presented. This technique has shown to be efficient and was validated using a PH model and a particular configuration based on a reduced WienerBose structure dynamic PA behavioral model. Later on, an innovative parallel model using preprocessing technique in the first branch and reduced Volterra series combined with subsampling techniques in the remaining ones was introduced. The extraction process used either an optimization of the resampling factor, based on function evaluations, or simulated annealing techniques, to reach the final result. This strategy and the model structure allowed improved identification performance, in terms of NMSE, in comparison to other simpler models. The main drawback is the increase in the number of parameters and

TABLE IV. Comparison NMSE SummarySimulated and Measured Signal

\begin{tabular}{lcrrrr}
\hline Model & $\begin{array}{c}\text { LM/NLM } \\
(\mathrm{dB})\end{array}$ & $\begin{array}{c}\text { LM } \\
(\mathrm{dB})\end{array}$ & $\begin{array}{c}\text { NLM } \\
(\mathrm{dB})\end{array}$ & $\begin{array}{r}\text { noM } \\
(\mathrm{dB})\end{array}$ & $\begin{array}{r}\text { Meas } \\
(\mathrm{dB})\end{array}$ \\
\hline LUT & -35 & -38 & -37 & -60 & -21 \\
Pre-WBose & -44 & -56 & -44 & -65 & -23 \\
PMRA & -48 & -56 & -48 & -65 & -29 \\
Branches & 3 & 1 & 4 & 1 & 5
\end{tabular}

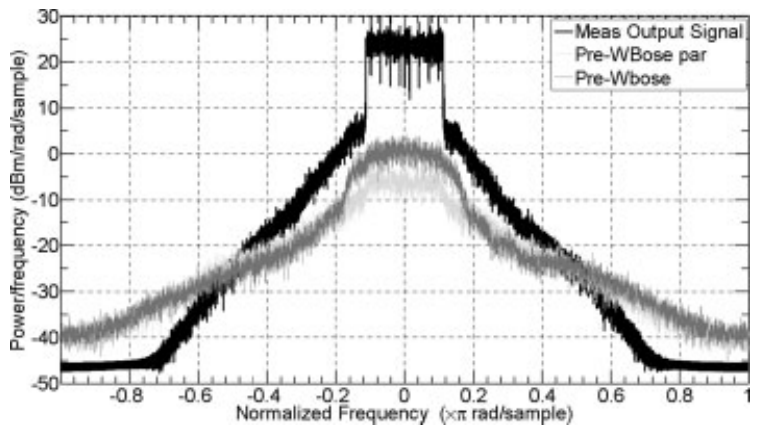

Figure 17. PSD of the measured output signal and residues of the PMRA (Pre-Wbose par) and the model with only preprocessing (Pre-Wbose).

simulation time when considering these modeling techniques. Results have shown that parallel models with different structures using subsampling in their subsequent branches can improve the identification performance and justify the inclusion of additional branches. Results also have confirmed that the accuracy of a PA behavioral model considering only one single branch is lower than considering preprocessing and resampling techniques. The importance of subband parallel models and optimization of the resampling factor was proven in terms of overall NMSE and spectrum in-band error improvement, showing an efficient reduction of the residue in the final estimation process.

\section{ACKNOWLEDGMENT}

This work was partially supported by TARGET_- "Top Amplifier Research Groups in a European Team," under contract IST-1-507893-NOE, www.target-net.org, CAPESBrazil, and the Spanish Government (MICINN) under project TEC2008-06684-C03-03.

\section{REFERENCES}

1. M.J. Korenberg, Identifying nonlinear difference equation and functional expansion representations: The fast orthoghonal algorithm, Ann Biomed Eng 16 (1988), 123-142.

2. C.P. Silva, A.A. Moulthrop, and M.S. Muha, Introduction to polyspectral modeling and compensation techniques for wideband communications systems, Automatic RF Tech Group Conf Digest 40 (2001), 1-15.

3. H. Ku, M. McKinley, and J.S. Kenney, Quantifying memory effects in RF power amplifiers, IEEE Trans Microwave Theory Tech 50 (2002), 2843-2849.

4. V.Z. Marmarelis, Nonlinear dynamic modeling of physiological systems, Wiley, New York, 2004. 
5. H. Ku and J.S. Kenney, Behavioral modeling of nonlinear RF power amplifiers considering memory effects, IEEE Trans Microwave Theory Tech 51 (2003), 2495-2504.

6. M. Isaksson, D. Wisell, and D. Ronnow, A comparative analysis of behavioral models for RF power amplifiers, IEEE Trans Microwave Theory Tech 1 (2006), 348-359.

7. O. Nelles, Nonlinear system identification, Springer, Berlin, 2001.

8. C.P. Silva, C.J. Clark, A.A. Moulthrop, and M.S Muha, Survey of characterization techniques for nonlinear communication components and systems, IEEE Aerospace Conf 1 (2000), 1-25.

9. J.C. Pedro and S.A. Maas, A comparative overview of microwave and wireless power-amplifier behavioral modeling approaches, IEEE Trans Microwave Theory Tech 53 (2005), 1150-1163.

10. P.L. Gilabert, M.E. Gadringer, G. Montoro, M.L. Mayer, D.D. Silveira, E. Bertran, and G. Magerl, Digital predistortion and OFDM clipping to enhance efficiency in power amplifiers, Int J RF Microwave Comput Aided Eng DOI 10.1002/mmce.20381.

11. S. Benedetto, E. Biglieri, and R. Daffara, Modeling and performance evaluation of nonlinear satellite links-A Volterra series approach, IEEE Trans Aerospace Electron Syst 15 (1979), 494-506.

12. D.D. Silveira and G. Magerl, Extraction and improvements of a behavioral model based on the WienerBose structure used for baseband Volterra Kernels estimation, Int Microwave Symp 1 (2007), 20072010.

13. A. Zhu, J.C. Pedro, and T.R. Cunha, Pruning the Volterra series for behavioral modeling of power amplifiers using physical knowledge, IEEE Trans Microwave Theory Tech 55 (2007), 813-821.

14. E. Ngoya and A. Soury, Modeling memory effects in nonlinear sub-systems by dynamic Volterra systems, IEEE Behavioral Modeling and Simulation Workshop, San José, 2003.

15. N. Le Gallou, E. Ngoya, H. Buret, D. Barataud, and J.M. Nebus, An improved behavioral modeling technique for high power amplifiers with memory, IEEE MTT Symp Digest 1 (2001), 983-986.

16. S. Mitra, A. Mahalonobis, and T. Saramaki, A generalized structural subband decomposition of FIR filters and its application in efficient FIR filter design and implementation, IEEE Tran Circ Syst II Analog Digital Signal Process 40 (1993), 363-374.

17. O. Hammi, S. Boumaiza, M. Jaidane, and F. Ghannouchi, Baseband digital predistortion using subband filtering technique, IEEE MTT-S Int Microwave Symp Digest 3 (2003), 1699-1702.

18. G. Cocchi and A. Uncini, Subband neural networks prediction for on-line audio signal recovery, IEEE Trans Neural Networks 13 (2002), 867-876.

19. P. Gilabert, D. Silveira, G. Montoro, M. Gadringer, and E. Bertran, Heuristic algorithms for power amplifier behavioral modeling, IEEE Microwave Wireless Compon Lett 17 (2007), 715-717.

\section{BIOGRAPHIES}

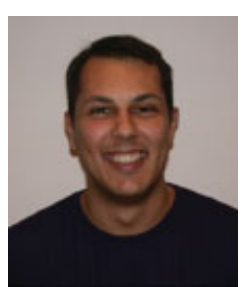

Daniel D. Silveira received the Bachelor degree in Electrical Engineering (2001) and Master in Telecommunications degree (2003) from Instituto Nacional de Telecomunicações (Brazil). In 2003 he joined the Institute for Electrical Measurement and Circuit Design at Vienna University of Technology, and in 2007 he received his Ph.D. from this university. Since January 2008, he is a researcher with Linear Equipamentos Eletrônicos (www.linear.com.br), Brazil, where he is involved with the development of new technologies for broadcast transmitters, including power amplifiers optimization, linearization, and passive components.

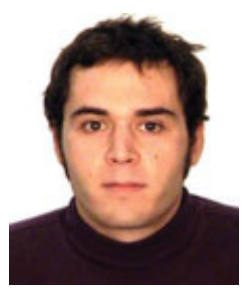

Pere Ll. Gilabert Pinal received the degree in Telecommunication Engineering from the Technical University of Catalonia (UPC) in 2002, and he developed his Master Thesis at the University of Rome "La Sapienza" with an exchange grant. $\mathrm{He}$ joined the department of Signal Theory and Communications in 2003 and received his Ph.D. from the UPC in 2008. His research is focused on system level linearization techniques for RF power amplifiers, digital signal processing for communications and circuit theory. He is currently lecturing on undergraduate courses at the Castelldefels School of Technology (EPSC) as Assistant Professor.

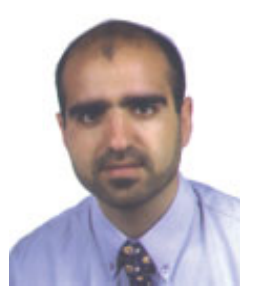

Pedro Lavrador received his Diploma and Ph.D. degrees in Electronics and Telecommunications Engineering from the Universidade de Aveiro, Aveiro, Portugal in 2001 and 2007 respectively. $\mathrm{He}$ is with the Telecommunications Institute (Aveiro) since October 2000. His main research interests are studying the impact of nonlinear effects in communication systems, and efficient simulation methods and models for the same kind of systems. He is now an invited assistant professor at Universidade de Aveiro. Mr. Lavrador had received three times, during his graduation studies, the prize for the students with the best grades of Engineering courses at the University of Aveiro. 


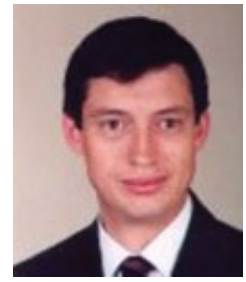

José C. Pedro received diploma, doctoral, and habilitation degrees in electronics and telecommunications engineering, from University of Aveiro, Portugal, in 1985, 1993, and 2002, respectively. From 1985 to 1993 he was Assistant Lecturer at University of Aveiro, and Professor since 1993. Currently, he is full-time Professor and Researcher at the same University. He is the author of Intermodulation Distortion in Microwave and Wireless Circuits (2003), and has authored or coauthored more than 150 articles in international journals and symposia, and is a reviewer and Associate Editor for the: IEEE MTT Transactions, IEEE MTT-IMS, and EuMiC. He has served the university as Coordinator of the Scientific Council and is now the Head of the Department. He received the Marconi Young Scientist Award in 1993; in 2000 the IEE Measurement Prize, and in 2007 he was elected as the Fellow of the IEEE for his contributions to the nonlinear distortion analysis of microwave devices and circuits, his main scientific interests.

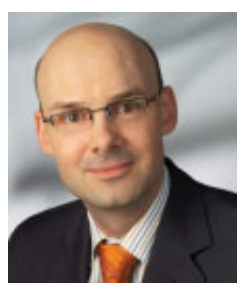

Michael E. Gadringer ( $\left.\mathrm{S}^{\prime} 03\right)$ received the Dipl.-Ing. from the Vienna University of Technology 2002, and is currently working toward his Ph.D. with a particular interest in amplifier linearization and behavioral modeling. Since January 2003 , he is a Research Assistant at the Institute of Electrical Measurement and Circuit Design at Vienna University of Technology. He has coedited the book: "RF Power Amplifier Behavioral Modeling" published by Cambridge University Press. He is currently involved in the design and optimization of amplifier measurement setups and device characterization using behavioral modeling techniques.

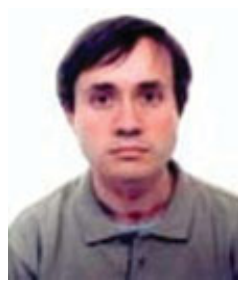

Gabriel Montoro was born in Barcelona (Spain). He received the M.S. degree in Telecommunication Engineering from the Technical University of Catalonia (UPC) in 1989 and did his final project in the area of Adaptive Control. He joined the department of Signal Theory and Communications (TSC) in 1991, where he is currently an Associate Professor. $\mathrm{He}$ received the Ph.D. degree of Telecommunications in 1996. His research interests include control, consumer, and communications electronics.

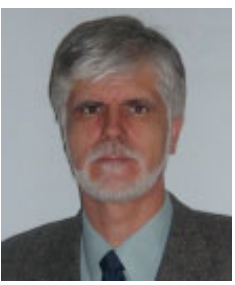

Eduard Bertran (M'91-SM'02) received the Engineer and Doctor Engineer degrees in Telecommunication Engineering, both from the Universitat Politècnica de Catalunya (UPC), in 1979 and 1985, respectively. He joined the department of Signal Theory and Communications (TSC) in 1987, where he is currently a full professor. He has been the head of studies of the TSC department and an associate dean in different telecommunications schools. His research interests include topics such as communication electronics, control, signal processing, and circuit theory, where he has produced several publications. He is a member of IFAC and a senior member of IEEE, and has collaborated in national and European research projects and teams.

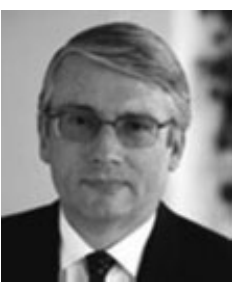

Gottfried Magerl received the Dipl.-Ing. and Dr. techn. degrees from the Vienna University of Technology, Austria, in 1972 and 1975, respectively. In 1990, he became University Professor of Microwave Engineering, and in 1998 he became the Head of the Institute of Electrical Measurement and Electronic Circuit Design. He spent the 1981-1982 academic year and the summer months of 1984 and 1986 at the University of Chicago and at Michigan State University, East Lansing, where he constructed high-resolution IR spectrometers based on microwave modulation of $\mathrm{CO}_{2}$ and $\mathrm{CO}$ lasers. In the following, his scientific interest shifted toward noncontacting measurement techniques. His current research interests concentrate on the development of highly efficient linear microwave power amplifiers for mobile communications. From 2004 to 2007, he was scientific coordinator of the European FP6 Network of Excellence called TARGET. Gottfried Magerl is author and coauthor of more than 200 contributions to international scientific journals and conferences. 